\title{
Novel Treatment Strategies for Neurological and Neurodegenerative Diseases
}

\author{
Simon Kaja* and Andrew J Payne
}

Department of Ophthalmology, School of Medicine, University of Missouri, Kansas City, USA, Tel: +1 (816) 235-6773; E-mail: kajas@umkc.edu

*Corresponding author: Simon Kaja, Assistant Professor, Department of Ophthalmology, School of Medicine, University of Missouri, Kansas City, USA, Tel: +1 (816) 235-6773; E-mail: kajas@umkc.edu

Received date: March 12, 2014 Accepted date: March 18, 2014, Published date: March 25, 2014

Copyright: ( 2014 Kaja S, et al. This is an open-access article distributed under the terms of the Creative Commons Attribution License, which permits unrestricted use, distribution, and reproduction in any medium, provided the original author and source are credited.

\section{Editorial}

Neurological and neurodegenerative diseases are a major burden not only to those individuals affected but also to families, caregivers and society as a whole. With life expectancy on the rise, we are facing new challenges of higher lifetime prevalence rates of neurodegenerative disorders and the associated increases in health care costs.

In recent years, preclinical and basic science efforts have resulted in significant advances in developing and testing novel preclinical models and assessing potential therapeutic agents. Many of these attempts have focused on neuroprotective strategies. The concept of neuroprotection is based on the assumption that neurodegenerative processes associated with disease and/or aging can be slowed, halted, or even reversed by therapeutic intervention. Common targets for neuroprotection include increased levels in oxidative stress, mitochondrial dysfunction, excitotoxicity, inflammatory processes, and protein aggregation.

Unfortunately however, most clinical trials on neuroprotection have failed despite promising preclinical data. One exception is the drug Memantine, FDA-approved as a cognitive enhancer in moderate to severe Alzheimer's disease. Does this mean that pursuing drug discovery targeted at neuroprotection for neurodegenerative and neurological disorders is a futile undertaking? Certainly not! Rather, several critical issues have to be considered to increase the chances of a successful translation of preclinical insights to meaningful clinical trials. These include the inherent limitations of in vitro studies, the suitability of preclinical animal models to mirror the various pathologies underlying human neurodegenerative diseases, and the lack of drug-likeness of most lead compounds. Furthermore, PD/PK studies are often ignored during early stages of drug discovery, although they may predict future challenges in the drug development pipeline.

For example, the therapeutic window of potential interventional strategies for ischemic stroke is of critical importance [1]. Intravenous administration of tissue plasminogen activator to reduce ensuing disability is indicated within the first three hours after a sudden ischemic stroke. However, most patients arrive at the ER between 3 and 6 hours after the first warning signs [2]. Future drug discovery efforts must take into account clinical reality and test more appropriate therapeutic time windows.
Similarly, neuroprotection strategies for ocular disorders require adequate behavioral readouts. In a clinical context, visual function is the sole determinant of successful therapy, however, many preclinical studies solely focus on molecular or cellular readouts to determine the effects of novel drug candidates [3]. Using the Opto Motry system (Cerebral Mechanics Inc.), functional vision and contrast sensitivity can accurately be determined in both mice and rats. Utilizing this technology we have reported impaired visual function in the DBA/2J mouse model for glaucoma. Critically, the reduced visual function was correlated with elevated intraocular pressure, a clinically relevant biomarker for glaucoma [4,5]. Future preclinical development must incorporate these available technologies, and focus on clinically relevant outcome parameters.

For this Special Issue we have solicited original research articles, topical reviews, and clinical case reports focusing on "Novel treatment strategies for neurological and neurodegenerative diseases".

On behalf of the Journal of Biology and Medicine and the Editorial Board, we would like to thank all authors for their contributions. We look forward to your future submissions on the promising and challenging aspects of the field of neurodegenerative and neurological disorders.

\section{References}

1. Xu SY, Pan SY (2013) The failure of animal models of neuroprotection in acute ischemic stroke to translate to clinical efficacy. Med Sci Monit Basic Res 19: 37-45.

2. Leon-Jimenez C, Ruiz-Sandoval JL, Chiquete E, Vega-Arroyo M, Arauz A, et al. (2013) Hospital arrival time and functional outcome after acute ischemic stroke: Results from the PREMIER study. Neurologia 121-127.

3. Payne AJ, Kaja S, Naumchuk Y, Kunjukunju N, Koulen P (2014) Antioxidant drug therapy approaches for neuroprotection in chronic diseases of the retina. Int J Mol Sci 15: 1865-1886.

4. Burroughs SL, Kaja S, Koulen P (2011) Quantification of deficits in spatial visual function of mouse models for glaucoma. Invest Ophthalmol Vis Sci 52: 3654-3659.

5. Kaja S, Naumchuk Y, Grillo SL, Borden PK, Koulen P (2014) Differential up-regulation of Vesl-1/Homer 1 protein isoforms associated with decline in visual performance in a preclinical glaucoma model. Vision Res 94: 16-23. 